Ana V. Ferreira
Vadim Volkov

Ana S. Abreu*

Nuno Azoia

Cláudia M. Botelho

Artur Cavaco-Paulo

Centre of Biological Engineering

(CEB), University of Minho,

Braga, Portugal
Research Article

\section{Orange IV stabilizes silk fibroin microemulsions}

Silk fibroin (SF) is a natural biopolymer that has been extensively studied in various applications due to its impressive mechanical properties and biocompatibility. Recently, SF-based particles have been proposed as controlled drug delivery systems. A new and efficient method to prepare SF microemulsions (SF-MEs) was developed by oil-in-water emulsions using high-pressure homogenization to promote emulsification. During SF-ME production, the secondary structure of SF changed to a more stable conformation (from random coil to $\beta$-sheets), thus allowing the formation of small and stable (140.7 $\pm 1.9 \mathrm{~nm}$; polydispersity index, 0.25) SF microparticles (SF-MPs). The efficiency of SF-MP formation was $60 \%$. Orange IV was used as a model compound for incorporation and release studies, although its incorporation into the SF-MEs significantly improved particle size and size distribution over at least $4 \mathrm{wk}$ compared to traditional stabilizers (e.g., poloxamer 407, transcutol, Tween 80, and SDS). This should be a call of attention when using dyes as model compounds since they can influence particle properties and lead to misinterpretation of the results. Orange IV showed an incorporation efficiency of $91 \%$ and a controlled release over time. Stable SF-MP formulations, further enhanced by orange IV incorporation, provide an innovative method with potential application in pharmaceutical development due to its associated high biocompatibility and release profile.

Keywords: High-pressure homogenizer / Microemulsions / Microparticles / Orange IV / Silk fibroin

Received: September 4, 2014; revised: November 26, 2014; accepted: January 13, 2015

DOI: $10.1002 /$ elsc.201400190

\section{Introduction}

Silk fibroin (SF) is largely exploited as a biomaterial due to its unique characteristics, namely, strength, excellent biocompatibility, good oxygen permeability, high thermal stability, slow biodegradation, and minimal inflammatory and immunogenic response [1-4]. This FDA-approved biopolymer [3,5] can be further processed into different materials, such as $3 \mathrm{D}$ scaffolds $[6,7]$, films $[7,8]$, electrospun fibers [7,9], and microspheres [7].

The primary structure of SF protein from Bombyx mori silkworm cocoons is characterized by the sequence [Gly-Ala-

Correspondence: Prof. Artur Cavaco-Paulo (artur@deb.uminho. pt), Centre of Biological Engineering (CEB), University of Minho, Campus of Gualtar, 4710-057 Braga, Portugal

Abbreviations: FTIR, FT infrared spectroscopy; HPH, high-pressure homogenizer; MEs, microemulsions; MPs, microparticles; PDI, polydispersity index; SF, silk fibroin; SF-MEs, silk fibroin microemulsions; SF-MPs, silk fibroin microparticles

*Current address: Ana S. Abreu, Institute of Polymers and Composites (IPC), Institute Nanostructures, Nanomodelling and Nanofabrication

(I3 N), University of Minho, Guimarães, Portugal
Gly-Ala-Gly-Ser $]_{n}$, which folds into a secondary structure of antiparallel $\beta$-sheets $[10,11]$. The SF stability is mainly dependent on the $\beta$-sheet content and its presence increases the interaction with different substances for a long period of time. This feature makes the use of SF suitable for drug delivery purposes [12,13].

One of the most commonly used methods for the production of protein-based particles is emulsification [14-16]. However, emulsions are nonequilibrium and nonspontaneous systems [17] and, therefore, its production requires a large input of energy or the addition of surfactants for stabilization [18-20]. Emulsification can be achieved using high-energy methods, in which intensely disruptive forces break the oil and water phases creating micro- and nanosized droplets $[12,17]$. The highenergy mechanical devices can be ultrasonicators, high-pressure homogenizers (HPHs), or microfluidizers [12, 17].

Previously, our research group was able to prepare BSA, HSA, and SF emulsions by the ultrasonic emulsification method [21-23]. However, the large dimensions of the resulting SF particles were inappropriate for drug delivery purposes [21-23]. In continuity of this work, it was necessary to optimize the emulsification process for the production of SF particles suitable for drug delivery. 


\section{Engineering}

In order to obtain stable particles that maintain their characteristics over time, it is important to choose and optimize the preparation method, as well as to add stabilizers into the formulation [19]. It is known that stabilizers prevent the system to revert into a lower-energy configuration of the thermodynamic equilibrium [19]. Polymeric surfactants are one of the most effective stabilizers, since they control and prevent flocculation and coalescence effects to occur [24].

Some of the most common surfactants used are poloxamer 407, Tween 80, transcutol, and SDS. Poloxamer 407 and Tween 80 are nonionic polymeric surfactants composed of poly(ethylene glycol) and poly(propylene glycol) [24,25], and polyoxyethylene [20] and sorbitan monooleate [26,27] chains, respectively. SDS, the most common anionic surfactant, is capable of adsorbing the oil-droplet surfaces within homogenizers [28]. Similar to poloxamer 407, SDS acts as a gelling agent by inducing selfassembly of SF into stable $\beta$-sheet structures [29], and like Tween 80 , it possesses an amphipathic structure, also being considered as a detergent [30]. Transcutol is not only a powerful stabilizing agent, but it is also a skin penetration enhancer [31], adding an extra value to cosmetic formulations. This short-chain alcohol is mainly used as an additive due its weak amphiphilic character, being considered as a cosurfactant [31-33].

To evaluate the efficiency of the particulate drug delivery systems, incorporation and release studies can be performed using dyes as model compounds [34-37]. The use of dyes facilitates the study of the release profile, as the dye can be qualitatively (visually) and quantitatively detected [34-37]. For instance, orange IV can be easily quantified by UV-Vis spectrophotometry at $240 \mathrm{~nm}[38,39]$. Herein, we present the development of SF microemulsions (SF-MEs) using an HPH in the absence or presence of different types of nonionic and neutral surfactants to enable efficient and safe drug delivery.

\section{Materials and methods}

\subsection{Materials}

Cocoons of Bombyx mori silkworms were kindly supplied by the CRA - Unitá di Ricerca di Apicoltura e Bachicoltura (Padova, Italy). All chemical reagents were of analytical grade and were purchased from Sigma-Aldrich (Spain). The dialysis tubing cellulose membrane (MWCO, 12-14 kDa) was from Sigma-Aldrich, and centrifuge filtration devices Amicon Ultra-15 (MWCO, $3 \mathrm{kDa}$ ) were from Millipore Corporation (Ireland).

\subsection{Methods}

\subsubsection{Preparation of regenerated SF}

SF stock solutions were prepared as previously described [40,41]. Briefly, raw silk from the $B$. mori silkworms cocoons was boiled three times in an aqueous solution of $\mathrm{Na}_{2} \mathrm{CO}_{3}(0.05 \%)$ for $30 \mathrm{~min}$ and washed several times with deionized water for complete sericin removal. The dried degummed silk was then dissolved in $9 \mathrm{M}$ lithium bromide ( $\mathrm{LiBr}$ ) for $3 \mathrm{~h}$ at $60^{\circ} \mathrm{C}$ and, after filtration, the resulting SF solution was dialyzed in cellulose tubing (MWCO, 12-14 kDa) against distilled and deionized water for at least 3 days at room temperature to remove the neutral salts and LiBr. All impurities were accounted in our work, as described in the cited procedures $[40,41]$.

The final solution of SF (termed as regenerated SF, RSF) was quantified at $595 \mathrm{~nm}$ by the Bradford method (Bio-Rad Laboratories) using SF as a standard protein. The calibration curve of SF for Bradford quantification (data not shown) was determined with several concentrations of SF, after weighting previously prepared and lyophilized SF protein.

\subsubsection{Preparation of SF-MEs}

SF-MEs were produced in an APV-2000 double-stage HPH (supplied from SPX Flow Technology, APV Manufacturing, Poland). Optimization was done at a preliminary analysis (data not shown), in which various parameters were evaluated, such as SF concentration (range $1-10 \mathrm{~g} / \mathrm{L})$, oil-in-water ratio $(10 / 90$, $20 / 80$, and 40/60), stabilizer concentration (range $0.5-10 \%$ of $\mathrm{w} / \mathrm{v}$ ), and number of homogenising cycles (range 2-28). The optimized formulations were produced with $80 \mathrm{~mL}$ of $10 \mathrm{~g} / \mathrm{L}$ of regenerated SF containing $1 \%$ of w/v of stabilizers (poloxamer 407 (poly[ethylene glycol]-block-poly[propylene glycol]-block-poly[ethylene glycol], $\left.\left(\mathrm{C}_{3} \mathrm{H}_{6} \mathrm{O}_{2} \mathrm{C}_{2} \mathrm{H}_{4} \mathrm{O}\right)_{n}\right)$, transcutol (di[ethylene glycol] ethyl ether, $\mathrm{C}_{6} \mathrm{H}_{14} \mathrm{O}_{3}$ ), Tween 80 (polyoxyethylene 80 sorbitan monooleate, $\mathrm{C}_{64} \mathrm{H}_{124} \mathrm{O}_{26}$ ) or SDS $\left(\mathrm{CH}_{3}\left(\mathrm{CH}_{2}\right)_{11} \mathrm{OSO}_{3} \mathrm{Na}\right)$, and $20 \mathrm{~mL}$ of $n$-dodecane that passed through two homogenizing devices connected in series, with a first stage at 580-600 bar and a second stage at 240-250 bar for 22 homogenization cycles.

Also, orange IV (sodium 4-[(E)-[4-(phenylamino)phenyl] diazeny]benzenesulfonate, $\mathrm{C}_{18} \mathrm{H}_{14} \mathrm{~N}_{3} \mathrm{NaO}_{3} \mathrm{~S}$ ) at $1 \%$ of w/v was added to the $\mathrm{SF}$ solution $(10 \mathrm{~g} / \mathrm{L})$. The mixture was gently shaken until no solid was observed in suspension and the subjected to homogenization. For incorporation studies, concentrations of 50,75 , and $100 \mu \mathrm{M}$ of orange IV were used.

\subsubsection{Characterization of SF-MEs}

2.2.3.1 Structural analysis by FT infrared spectroscopy (FTIR) The FTIR spectra of the lyophilized SF solution and the produced SF-MEs were obtained using Jasco FT/IR 4100 spectrometer equipped with attenuated total reflectance accessory. The infrared spectra were recorded in transmission mode between 600 and $4000 \mathrm{~cm}^{-1}$ by accumulation of 128 scans with a resolution of $8 \mathrm{~cm}^{-1}$. Gaussian deconvolution of Amide I band region (wavenumber between 1600 and $1700 \mathrm{~cm}^{-1}$ ) was analyzed using OriginPro 8.5 software (OriginLab Corporation, MA, USA).

\subsubsection{Size and surface charge evaluation}

Using the NANO ZS Malvern Zetasizer equipment (Worcestershire, UK), SF-MPs were characterized regarding their mean diameter, size distribution and polydispersity index (PDI) by dynamic light scattering, and zeta-potential values by laser Doppler anemometry. This characterization was performed immediately after the emulsification process and at different time points over $2-5 \mathrm{wk}$, the samples having been stored at $4^{\circ} \mathrm{C}$. 


\subsubsection{SF-MP efficiency of formation}

Efficiency of formation of the SF-MPs was determined using Eq. (1). For that, SF-MEs were centrifuged $(10976 \times g, 60$ $\mathrm{min}$ ) in order to separate the free SF protein from the MPs, the resulting supernatant of each sample being collected and quantified by Bradford method $(n=3)$.

$$
\begin{aligned}
& \text { Formation Efficiency }(\%)= \\
& \frac{[\text { Protein }] \text { initial }-[\text { Protein }] \text { final }}{[\text { Protein }] \text { final }} \times 100
\end{aligned}
$$

2.2.3.4 Encapsulation efficiency of orange IV into the SF-MPs Orange IV loaded SF-MPs were centrifuged $(5000 \times g, 45 \mathrm{~min})$ using centricon tubes $(100 \mathrm{kDa})$, and the resulting filtrate was quantified $(n=3)$ at $240 \mathrm{~nm}$ using UV spectrometry and a calibration curve of orange IV standards (data not shown). The encapsulation efficiency (\%) of orange IV into the MPs was determined using Eq. (2).

$$
\begin{aligned}
& \text { Encapsulation Efficiency }(\%)= \\
& \frac{[\text { Orange IV }] \text { initial }-[\text { Orange IV }] \text { final }}{[\text { Orange IV }] \text { final }} \times 100
\end{aligned}
$$

\subsubsection{In vitro orange IV release studies from SF-MPs} Orange IV loaded SF-MPs and control samples (total volume of $2 \mathrm{~mL}$ ) were placed in a dialysis bag (cellulose membrane, MWCO $12-14 \mathrm{kDa}$ ) that was sealed and immersed in $10 \mathrm{~mL}$ of deionized water, with continuous magnetic stirring (200 rpm) at room temperature for 6 days. At determined time intervals, dialysis volume was withdrawn, quantified $(n=3)$, and replaced with deionized water. Free orange IV $(87 \mu \mathrm{M})$ dissolved in 10 $\mathrm{g} / \mathrm{L}$ of SF solution and in deionized water was also placed in a dialysis bag and used as control.

\subsubsection{3D simulations}

The 3D simulations were performed with the GROMACS 4.6 [42] package using the GROMOS force field. Initially, the protein was centered in the box and the orange IV molecules were randomly distributed in the simulation box. The system size was chosen according to the minimum image convention, taking into account a cutoff of $1.4 \mathrm{~nm}$. The bonds lengths of the proteins were constrained with LINCS [43] and those for water with SETTLE [44]. Nonbonded interactions were calculated using a twin-range method, with short and long range cutoffs of 0.8 and $1.4 \mathrm{~nm}$, respectively. Neighbor searching was carried out up to $1.4 \mathrm{~nm}$ and updated every five steps. A time step of integration of $2 \mathrm{fs}$ was used. A reaction field correction for the electrostatic interactions was applied using a dielectric constant of 54 [45]. The single point charge model [46] was used for water molecules. The initial systems were energy minimized for 2000 steps using the steepest descent method, with all heavy atoms harmonically restrained using a force constant of $1000 \mathrm{~kJ} / \mathrm{mol}$ $\mathrm{nm}^{2}$. The systems were initialized in the canonical ensemble (NVT) for $50 \mathrm{~ns}$, with all heavy atoms harmonically restrained using a force constant of $1000 \mathrm{~kJ} / \mathrm{mol} \mathrm{nm} \mathrm{n}^{2}$. The simulation was then continued for $50 \mathrm{~ns}$ in the isothermal-isobaric ensemble (NPT), with the heavy atoms harmonically restrained with the same force constant. Finally, for allowing the equilibration of the system properties, the simulations were further extended in the NPT ensemble with positional restrains applied to the $\mathrm{C} \alpha$ atoms. Pressure control was implemented using the Berendsen barostat [47], with a reference pressure of 1 bar, 0.5 ps of relaxation time, and isothermal compressibility of $4.5 \times 10^{5}$ bar. Temperature control was set using the $\mathrm{V}$ rescale $[47,48]$ thermostat at $300 \mathrm{~K}$. The protein and the solvent molecules were coupled in separated heat baths, with temperatures coupling constants of $0.025 \mathrm{ps}$ in the first two initialization steps and with $0.1 \mathrm{ps}$ for the rest of the simulations. The simulation was carried out for 10 ns.

\section{Results and discussion}

\subsection{SF-ME formation}

The SF microparticles (SF-MPs) were produced by high-energy emulsification using high-pressure homogenization method. In this process, the ME formation and, consequently, the MP assembly are owed to the action of high-energy disruptive forces, namely, the hydraulic shear, intense turbulence, and cavitation [17]. Due to these forces' involvement, along this process SF protein may suffer conformational changes [12, 17, 49].To better understand the mechanisms involved in the HPH emulsification, the SF secondary structure of the developed SF-MEs was evaluated by FTIR; the obtained results are presented in Fig. 1.

According to Fig. 1A, the SF-MPs presented higher signal for $\beta$-sheets, suggesting this secondary structure is dominant in this sample, wherein the SF solution (before HPH emulsification) presented high signal for random coil in amide I. Since Amide I band (1600 and $1700 \mathrm{~cm}^{-1}$ ) is considered the most prominent and sensitive vibrational band $[50,51]$, and its spectrum is not significantly affected by side chain vibration [52], deeper analysis was conducted through Gaussian deconvulation. The results obtained according to the deconvulation of Amide I, namely, SF content in $\beta$-sheets, $\beta$-turns, random coil, and $3_{10}$-helix, are presented in Fig. 1B. As it can be seen in Fig. 1B, SF subjected to the HPH process increased the percentage of $\beta$-sheets. The native conformation of SF has approximately $40 \%$ of $\beta$-sheets, while SF-MEs show approximately $65 \%$ of $\beta$-sheet content. On the other hand, the random-coil content slightly decreased from $\sim 25$ to $\sim 21 \%$, respectively. These results suggest that the forces involved in the HPH method lead SF to acquire a more stable conformation, by increasing the crystalline structure of $\beta$-sheets.

The results obtained are in accordance with the literature $[12,21,23,53]$, where it is described that SF particles prepared by mechanical mixing and self-assembly process are predominantly composed by $\beta$-sheets. The formation of $\beta$-sheets increases encapsulation efficiency of small molecules and enables a tight control of the release kinetics $[12,13]$. Therefore, the developed system is ideal for biomedical applications, such as drug delivery.

The use of emulsification processes in SF solutions, associated to the enrichment of $\beta$-sheet content in the protein, usually promotes the formation of spherical particles $[12,19,54]$. 


\section{Engineering}

www.els-journal.com in Life Sciences

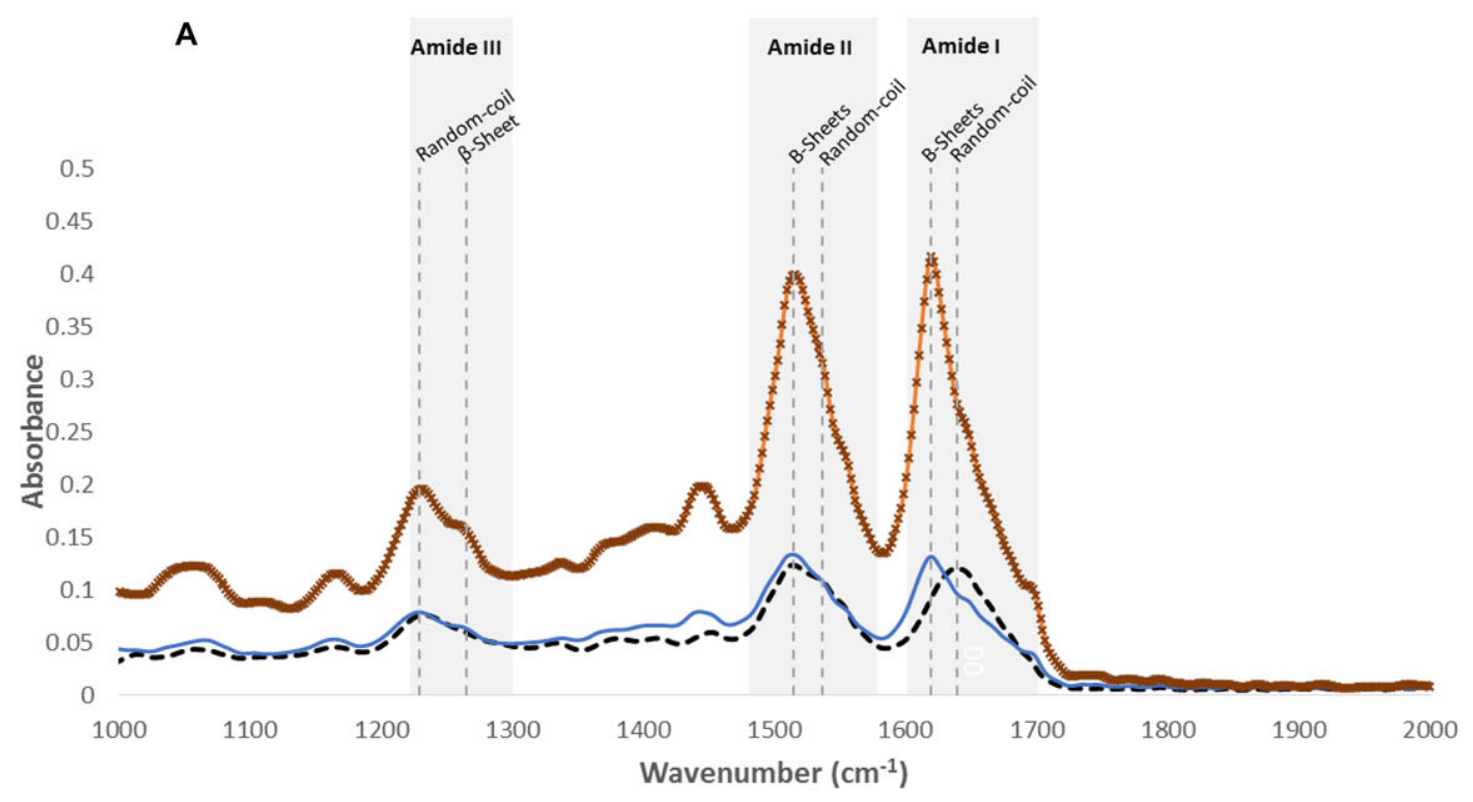

--- Silk Fibroin Solution at $10 \mathrm{~g} / \mathrm{L}$ (before HPH emulsification process)

- Silk Fibroin Microparticles (after HPH emulsification process)

—— Silk Fibroin Microparticles incorporated with $100 \mu \mathrm{M}$ of Orange IV (after HPH emulsification process)

B

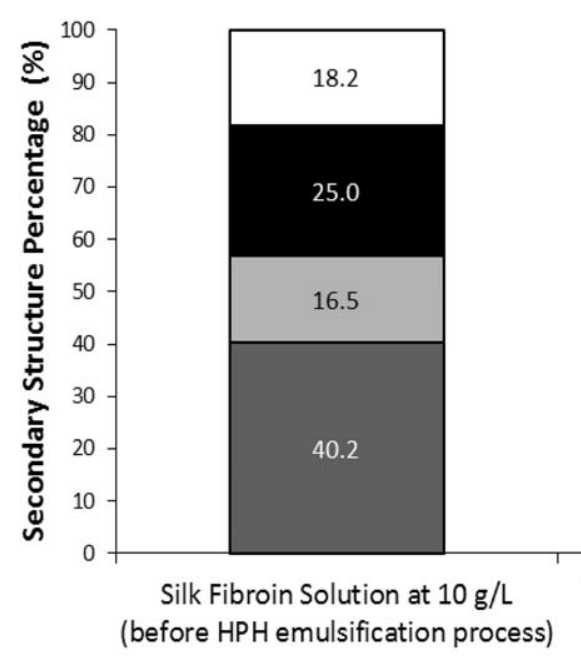

(before HPH emulsification process)

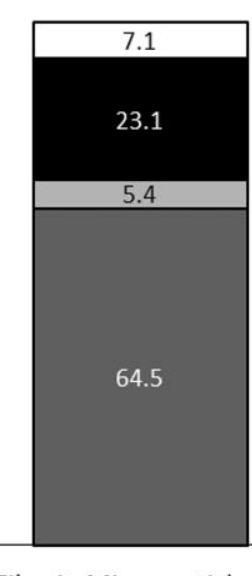

Silk Fibroin Microparticles (after $\mathrm{HPH}$ emulsification process)

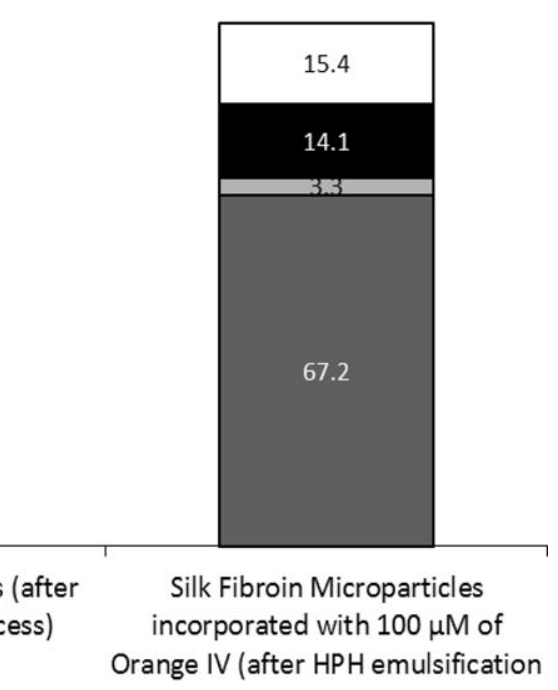

process)

\section{Material type}

$\square \beta$-sheet $\quad \square \beta$-Turn $\quad$ Random-coil $\quad \square 3[10]$ Helix

Figure 1. (A) FTIR spectra of SF protein before and after HPH emulsification. (B) Results from the deconvulation of amide I region (1600 and $1700 \mathrm{~cm}^{-1}$ ) of SF protein before and after $\mathrm{HPH}$ emulsification process.

Particularly, Silva et al. [21] have observed by STEM the morphology of SF-MPs produced by sonochemical emulsification and described a spherical shape with smooth surfaces. The authors also highlight that particles with spherical morphology provide minimum contact with the aqueous environment and longer diffusion transport, thus allowing efficient entrapment of drugs and higher controlled release over time [21].

Despite that $n$-dodecane can cause cytotoxicity (according to manufacturer's product data sheet), previous work done by our research group showed that only high ratios of $n$-dodecane 


\section{Engineering}

in Life Sciences

www.els-journal.com
Table 1. Yield of SF-MPs produced by high-pressure homogenization according to the efficiency of formation in percentage

\begin{tabular}{ll}
\hline Sample & $\begin{array}{l}\text { SF-MP yield efficiency } \\
\text { of formation (\%) }\end{array}$ \\
\hline SF-MPs (control) & $60.1 \pm 3.0$ \\
SF-MPs with Tween 80 & $26.5 \pm 4.8$ \\
SF-MPs with Transcutol & $60.9 \pm 3.3$ \\
SF-MPs with SDS & $76.3 \pm 2.0$ \\
SF-MPs with Orange IV & $74.9 \pm 3.5$ \\
\hline
\end{tabular}

(above 40\%) were considered toxic in the human cell line RKO (unpublished data and $[21,23]$ ). Although a proven absence of toxicity can only be evaluated with direct tests to evaluate the SF-ME effect on cells, we may predict that the formulations produced in this study should be biocompatible and will not lead to cytotoxicity due to $n$-dodecane. SF-MPs produced by $\mathrm{HPH}$ emulsification with an efficiency formation of $60 \%$ (Table 2) presented a small mean diameter $(140.7 \pm 1.9 \mathrm{~nm})$ with an acceptable PDI of 0.25 (Fig. 2A and B).
Table 2. Orange IV incorporation yield into SF-MPs produced by high-pressure homogenization according to formation and encapsulation efficiencies in percentage

\begin{tabular}{lll}
\hline & $\begin{array}{l}\text { SF-MPs yield } \\
\text { formation } \\
\text { efficiency (\%) }\end{array}$ & $\begin{array}{l}\text { SF-MPs } \\
\text { encapsulation } \\
\text { efficiency (\%) }\end{array}$ \\
\hline $\begin{array}{l}\text { SF-MPs with } 50 \mu \mathrm{M} \text { of } \\
\text { Orange IV }\end{array}$ & $74.9 \pm 3.5$ & $26.5 \pm 0.7$ \\
$\begin{array}{l}\text { SF-MPs with } 75 \mu \mathrm{M} \text { of } \\
\text { Orange IV }\end{array}$ & $72.3 \pm 2.1$ & $83.1 \pm 1.4$ \\
$\begin{array}{l}\text { SF-MPs with } 100 \mu \mathrm{M} \text { of } \\
\text { Orange IV }\end{array}$ & $66.9 \pm 2.1$ & $91.4 \pm 0.1$ \\
\hline
\end{tabular}

\subsubsection{SF-ME stability studies}

Despite the successful formation of SF-MPs, the size and size distribution of the obtained MPs were stable only for $2 \mathrm{wk}$. As presented in Fig. 2A and B, SF-MP size and size distribution values increased significantly at the second week. These findings clearly demonstrate the need to incorporate a stabilizer into
A

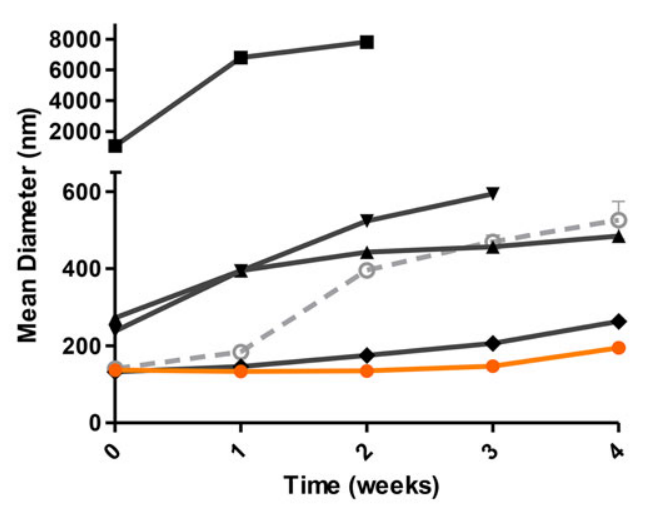

SF-NPs (control)

SF-NPs with Tween 80

\section{B}

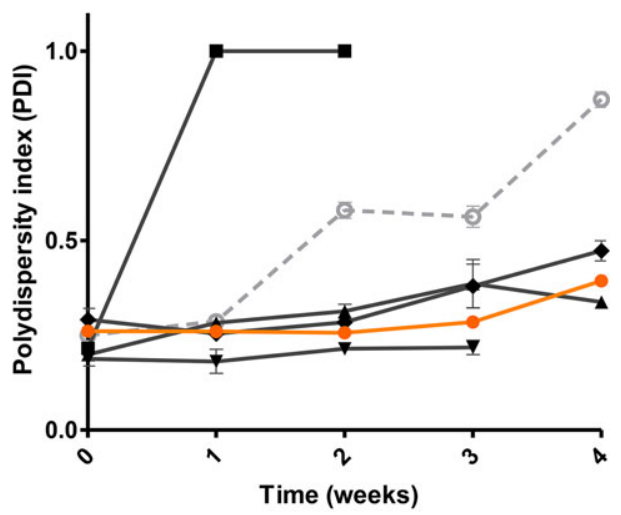

SF-NPs with Poloxamer 407

SF-NPs with Orange IV

C

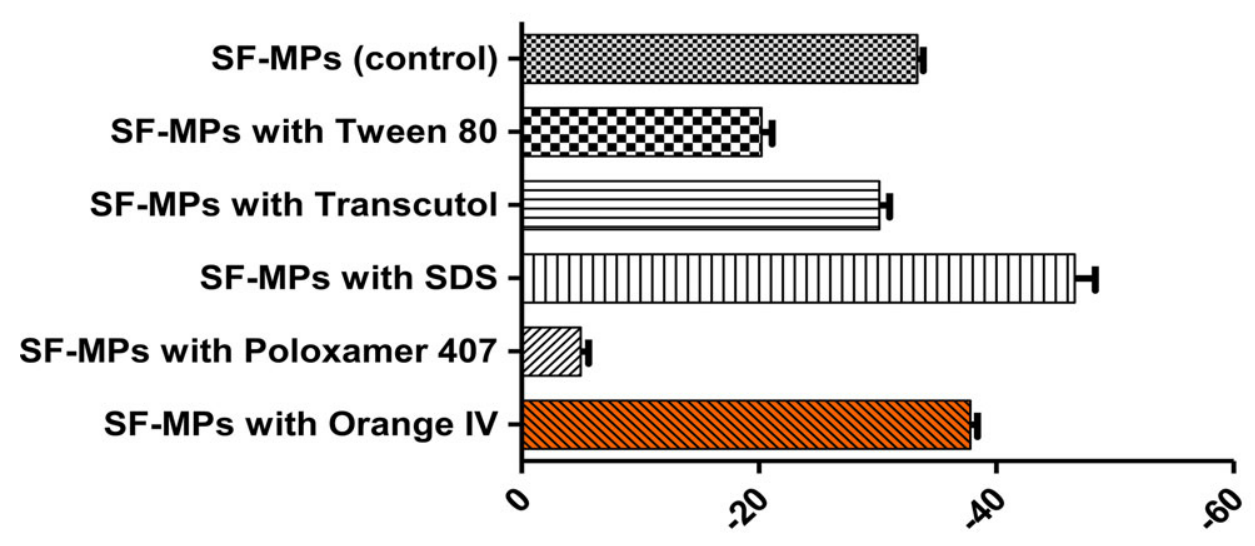

Zeta potential $(\mathrm{mV})$

Figure 2. Evaluation of the stability of SF-MPs produced by high-pressure homogenization according to (A) mean diameter in nanometers $(\mathrm{nm})$; (B) PDI values, over several weeks; and $(C)$ zeta-potential determination in millivolts $(\mathrm{mV})$ at the time of production. Error bars represent means \pm SD of three independent experiments. 


\section{Engineering}

www.els-journal.com

\section{in Life Sciences}

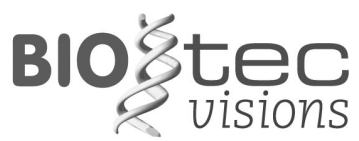

www.biotecvisions.com

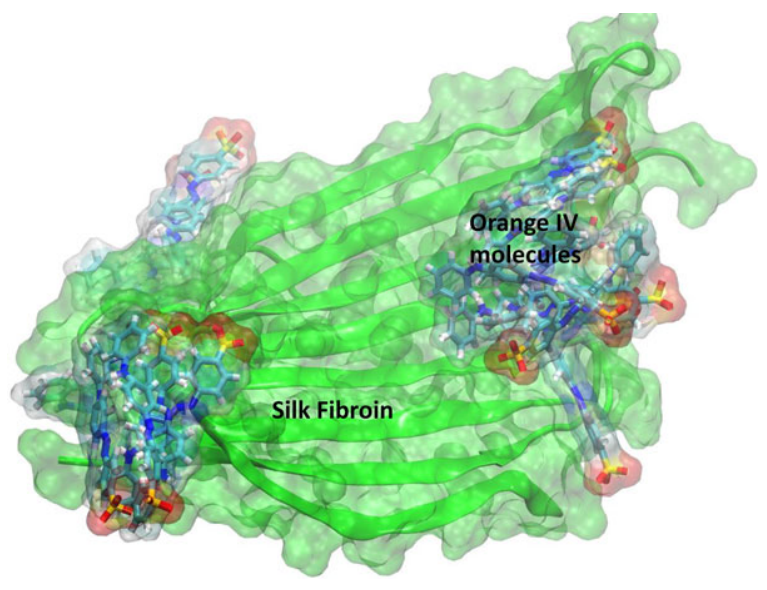

Figure 3. Representation of SF in $80 / 20 \mathrm{v} / \mathrm{v}$ mixture of water $/ \mathrm{n}$ dodecane with incorporation of orange IV molecules. SF protein is presented in green, evidencing the $\beta$-sheets, and the sticks structures represent 17 molecules of orange IV interacting with the SF structure. Portion of $n$-dodecane is not shown to facilitate visualization.

the SF-ME formulation. Therefore, poloxamer 407, Tween 80, transcutol, and SDS were tested.

As shown in Fig. $2 \mathrm{~A}$ and $\mathrm{B}$, the incorporation of the surfactants Tween 80 , SDS, and transcutol into the formulation increased the stability over time of the SF-MP mean diameter and PDI in comparison to the SF-MPs without stabilizers (control). The effect of these compounds on the SF-MP stability was further reflected on their high absolute zeta-potential values (Fig. 2C). In detail, our interpretation of the zeta-potential values was done according to the Riddick's Zeta-Meter $[57,58]$. As described in the literature $[55,56]$, higher absolute values of zeta potential (over $\pm 25 \mathrm{mV}$ ) predict the formation of stable emulsions. Charge with high absolute values (negative or positive) increases the electrostatic repulsion of other molecules, and consequently prevents unstable events such as agglomeration and flocculation to occur $[55,56]$. However, the stability of a suspension cannot be estimated only by zeta-potential measurements $[57,58]$. Hence, in our work, this parameter was used only as a complemental indicator, whereas size and PDI measurements over time were more effective in the assessment of the formulations' stability.

Regarding poloxamer 407, its addition into the formulation did not significantly improve the SF-MP properties in terms of size (Fig. 2 A) and size distribution (Fig. 2B) over time, this further being corroborated by the low zeta-potential absolute values (Fig. 2C). These results were unexpected, since previous studies showed the high stability improvement of BSA MEs [59]. We, then, suggest that the inability of poloxamer 407 to stabilize the SF-MPs might be related to the number of propylene oxide chains present in the formulation $(0.33 \%)$. At this concentration, the number of propylene oxide chains may not be enough to effectively influence the particles' stability [24]. Therefore, it is predictable that an increase of poloxamer 407 concentration should enhance the MP stability [24]. However, incorporation of poloxamer 407 into the formulation at a higher concentration is not advisable since this compound is considered by FDA as slightly irritant [25]. Therefore, poloxamer 407 was considered a nonefficient stabilizer for SF-MPs, and consequently discarded from all formulations.

Formulations with transcutol presented the smallest variation in the mean diameter and PDI values (Fig. 2A and B). These results suggest that transcutol is involved in SF-MP stabilization by inducing the deposition of SF molecules around the oil [32, 33]. Besides the ability of transcutol to stabilize the SF-MPs, this compound is known to enhance drug permeation through skin/epidermis, making it suitable for topical and transdermal delivery applications [31].

SDS addition to the formulation also showed great stabilizing effect on the SF-MPs, this being more evident in the PDI values (Fig. 2B) and in the zeta-potential measurements (Fig. $2 \mathrm{C})$. In fact, the highest SF-MP formation efficiency $(\sim 76 \%)$ was obtained using SDS (Table 1). These results suggest that the gelling capacity of SDS leads SF protein to be organized around oil droplets, creating MPs with a stronger conformation [29]. According to Wu et al.'s studies, SDS prevents coalescence and flocculation to occur [29]. As described by these authors, high SDS concentration leads to strong hydrophobic forces and electrostatic effect triggering SF to self-assemble into stable $\beta$-sheets [29].

\subsubsection{Orange IV as stabilizer of SF-MPs}

Although not considered a typical surfactant, in this work, we assessed the effect of orange IV used as a model compound for incorporation studies, on the stabilization of the SF-MP formulation. According to Fig. 2A and B, the incorporation of orange IV enhanced the stabilization of the particles over time. Once again, this result was corroborated by the high absolute value of zeta potential (Fig. 2C). Additionally, the incorporation of orange IV increased the efficiency of SF-MP formation to 75\% (Table 1).

To our knowledge, this is the first time that this stabilizing ability of orange IV is reported. The orange IV ability to stabilize the SF-MPs is probably due to the establishment of hydrophobic interactions and van der Waals forces with SF protein by the azo $(\mathrm{N}=\mathrm{N})$ and sulfonic groups $(\mathrm{S}=\mathrm{O})[38,60]$. In addition, this stabilization effect may also be related to the dye ability to increase gelation of SF solutions, since gelation increases the emulsion stability over time $[60,61]$. A similar phenomenon was suggested by Xiao et al. [60], corroborating our hypothesis that the stabilization effect of orange IV is related to the ability of SF to adsorb dyes. As an acidic dye, orange IV dissolution in the SF solution decreases $\mathrm{pH}$ and causes SF protonation, reducing the repulsion and consequently enabling the SF-MP stabilization $[60,61]$. This is also, validated by Xiao et al.'s studies, in which they demonstrated that the $\mathrm{pH}$ highly influences the interaction effects between SF protein and dyes [60].

Our FTIR results of SF-MPs loaded with $1 \%$ of orange IV (Fig. 1B) corroborated the findings that the incorporation of the dye stabilizes the SF-MP structure. The dyed particles have the highest content of $\beta$-sheets and the lowest content of random-coil structure compared to SF solution and SF-MPs without orange IV. These results suggest that the maintainability of SF-MP size and size distribution might be related to the structural interactions between orange IV and SF. To illustrate 


\section{Engineering}

in Life Sciences

www.els-journal.com

Eng. Life Sci. 2015, 15, 400-409

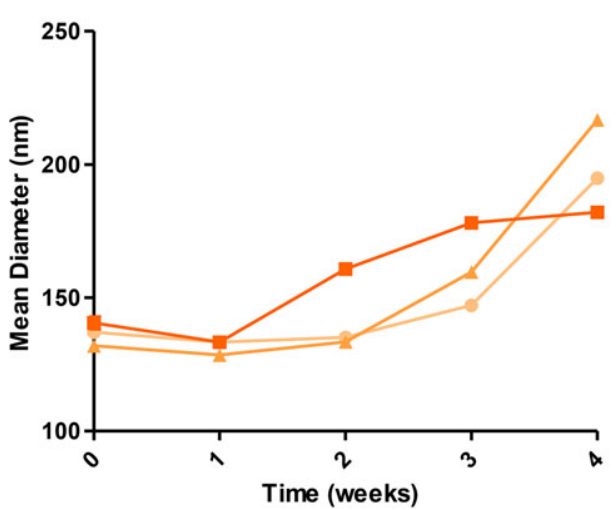

B

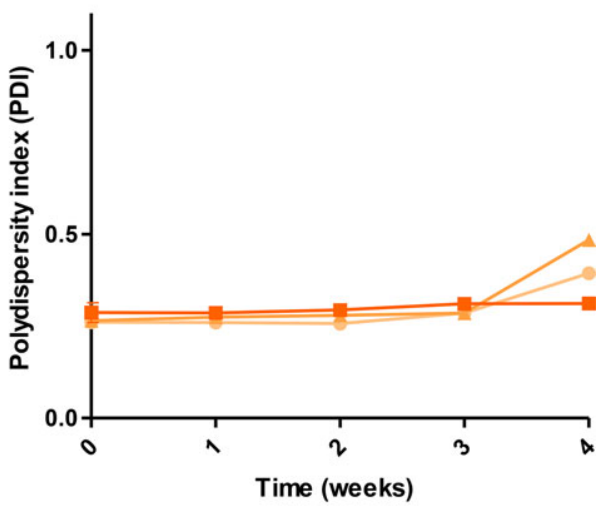

SF-NPs with $50 \mu \mathrm{M}$ Orange IV - - SF-NPs with $100 \mu \mathrm{M}$ Orange IV

SF-NPs with $75 \mu \mathrm{M}$ Orange N

C

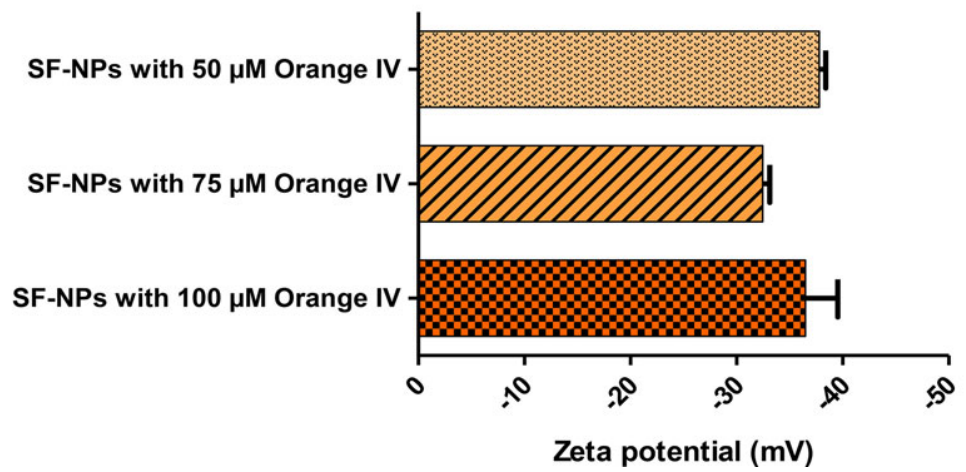

Figure 4. Evaluation of the stability of orange IV loaded SF-MPs, produced by highpressure homogenization, according to (A) mean diameter in nanometers $(\mathrm{nm})$; (B) PDI values, over several weeks; and (C) zeta-potential determination in millivolts $(\mathrm{mV})$ at the time of production. Error bars represent means \pm SD of three independent experiments.

our discussion, a representative image (Fig. 3) of the orientation of SF protein and orange IV molecules in an ME was designed to better understand the interaction between the two compounds. In this simulation, due to the high dimension $(\mathrm{MW}, 39 \mathrm{kDa})$ and characteristic repetitive domains ([Gly-Ala-Gly-Ala-Gly-Ser $]_{n}$ ) of SF protein, only a fragment of the SF repetitive arrangement is demonstrated. The orange IV molecules seem to have the ability to interact with the SF protein, leading to their entrapment in the protein structure. However, this behavior is somewhat unclear to us, and this system should be subjected to deeper studies. The combination of these results with the data concerning size measurements, PDI, and zeta potential support the previously stated stabilizing effect of orange IV on SF-MPs.

\subsection{Orange IV as a model compound for incorporation and release analysis}

Orange IV dye can be used as model for incorporation and drug release studies. In order to determine the maximum concentration of the dye to be incorporated without disturbing the stability of the MPs, several concentrations of orange IV were tested. In these assays, no additional stabilizers were added to the formulations.
Considering the results shown in Fig. 4A and B and Table 2, the maximum concentration of orange IV that could be optimally incorporated into the SF-MPs is $100 \mu \mathrm{M}$. At this concentration, we were able to obtain small particles $(\sim 140 \mathrm{~nm}$ of diameter) with an acceptable size distribution (PDI of 0.3 ) and with high efficiencies of formation and encapsulation (67 and $91 \%$, respectively). In addition, zeta-potential results (Fig. 4C) corroborated the sample stability by the measured high absolute zeta-potential value $(\sim-35 \mathrm{mV})$.

\subsubsection{Orange IV release profile from SF-MPs}

The release profile of orange IV incorporated into the SF-MPs was accessed by dialysis over 6 days (Fig. 5). Controls were performed dissolving the dye in water and in SF solution.

As it can be seen in Fig. 5, the free dye shows a fast diffusion rate in the first $18 \mathrm{~h}, 78.0 \pm 1.4 \%$ being recovered in the dialysis water. Then it follows a slower rate, with $92.6 \pm 4.8 \%$ of the dye being measured after $48 \mathrm{~h}$, and on the sixth day of dialysis $99.6 \pm$ $0.1 \%$ of the initial amount was quantified. The release of orange IV from SF-MPs follows the same trend although with lower amount of dye being recovered on the dialysis water. Hence, we measured a fast release of $39.4 \pm 0.9 \%$ during the first $18 \mathrm{~h}$, which increased around $10 \%$ after $48 \mathrm{~h}$ to an orange IV release of $48.2 \pm$ $0.9 \%$. At this time point, the formulation stabilized and no more 


\section{Engineering}

www.els-journal.com

\section{in Life Sciences}

Eng. Life Sci. 2015, 15, 400-409

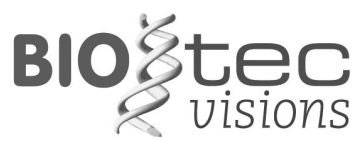

www.biotecvisions.com

— free Orange IV solution (control)

_free Orange IV dissolved in SF solution (control)

-Orange IV incorporated in SF-MPS

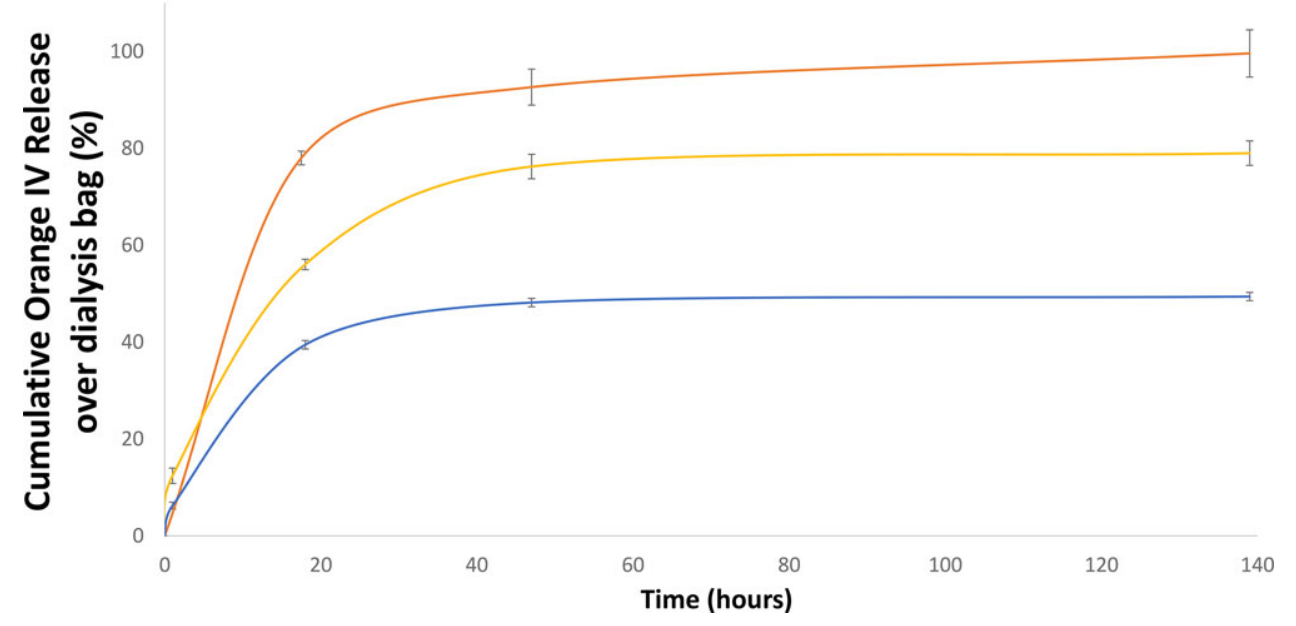

Figure 5. Orange IV release studies from SF-MPs incorporated with $100 \mu \mathrm{M}$ of orange IV. Orange IV dissolved in water and in regenerated silk fibroin (RSF) solution was used as control. Error bars represent means \pm SD of three independent experiments.

dye was released ( $49.4 \pm 0.9 \%$ on day 6$)$. As suggested previously (Fig. 3), orange IV is incorporated in the SF-MPs by interaction with SF $\beta$-sheets. Thus, it is expected that a fraction of the dye should be held into the MPs, and will only be released after the disruption of the particles. This assumption is also supported by the fact that, in the presence of SF in solution (Fig. 5), a fast release of $56.0 \pm 1.1 \%$ was measured after $18 \mathrm{~h}$ of incubation and the maximum of orange IV recovery was reached at $48 \mathrm{~h}(76.2 \pm$ $2.5 \%)$ without any further increase in the diffusion over 6 days of incubation $(79.0 \pm 2.5 \%)$. That is, SF itself has the ability to hold $20 \%$ of the dye, data that go in agreement with Xiao et al.'s findings of SF adsorption ability [60]. Additionally, these results also agree with previous suggestions about the capacity of orange IV in increasing SF gelation $[60,61]$.

The release of the incorporated orange IV from the SF-MPs was 1.5 and 2 times slower than the release of the dye from diffusion in the presence and absence of SF, respectively. Hence, the SF-MPs produced by HPH emulsification can incorporate and retain the orange IV in their structure and enable a slower and controlled release over time. Taking into account the previous results that orange IV possesses a greater stabilizer effect than traditional stabilizers, it is important to consider its use as model compound for drug incorporation and release studies, as its addition to the formulation is not inert. Our findings show that orange IV can influence the particles properties and can lead to misinterpretations of the results. The phenomenon described in this work is important as it can be considered as an innovative method to control molecules entrapment and release in formulations, since the presence of orange IV in SF-MPs yields stable particles over 3 wk (Fig. 2A and B).

\section{Concluding remarks}

Along the SF-ME production by HPH emulsification, the SF secondary structure changed from random coil to $\beta$-sheets. As the presence of $\beta$-sheets confers a more stable conformation, it is possible to produce small and stable SF-MPs $(140.7 \pm 1.9 \mathrm{~nm}$; PDI of 0.25 ) with a formation efficiency of $60 \%$.

Even so, the stability of SF-MP size and size distribution over time can be further improved by the incorporation of surfactants, such as transcutol. The incorporation and release studies using orange IV dye allowed us to discover a new application. According to our knowledge, this is the first time that the stabilizing effect of orange IV is described in SF-MPs. In our work, it is suggested that this effect might be related to orange IV azo $(\mathrm{N}=\mathrm{N})$ and sulphonic groups $(\mathrm{S}=\mathrm{O})$ that interact with $\mathrm{SF}$ protein, as illustrated in $3 \mathrm{D}$ simulation. These findings draw attention and concern the use of dyes as model compounds for incorporation and drug release studies, since the addition of dyes to formulations can influence the particles properties and mislead the interpretation of results.

The biocompatibility, stability, and controlled entrapment and release of these new developed SF-MPs have enhanced potential for biomedical applications, such as drug delivery with controlled release.

\section{Practical application}

The highlights of this work are as follows:

- A microemulsion (ME) of silk fibroin (SF) can be assembled by oil-in-water emulsification using high-pressure homogenization.

- The $\beta$-sheet content of SF protein increases during the ME production process.

- Orange IV has a strong stabilizing effect on SF-MEs.

The new preparation technique of SF-MEs presented here could be easily scaled up. SF is biocompatible, and the prepared MEs are stable and effective in cargo release. Therefore, the SF-MEs could be applied as a drug delivery system. 
The authors thank the FCT Strategic Project PEst-OE/EQB/ LA0023/2013 and the project "BioHealth - Biotechnology and Bioengineering Approaches to Improve Health Quality, "Ref. NORTE07-0124-FEDER-000027, cofunded by the Programa Operacional Regional do Norte (ON.2 - O Novo Norte), QREN, FEDER. We would also like to acknowledge CRA - Unitá di Ricerca di Apicoltura and Bachicoltura Padova (Italy) for the supply of B. mori silkworm cocoons, and Andreia Vasconcelos for technical support.

The authors have declared no conflict of interest.

\section{References}

[1] Ak, F., Oztoprak, Z., Karakutuk, I., Okay, O., Macroporous silk fibroin cryogels. Biomacromolecules 2013, 14, 719-727.

[2] Altman, G., Diaz, F., Jakuba, C., Calabro, T. et al., Silk-based biomaterials. Biomaterials 2003, 24, 401-416.

[3] Cao, Y., Wang, B., Biodegradation of silk biomaterials. Int. J. Mol. Sci. 2009, 10, 1514-1524.

[4] Jin, H., Liu, X., Yin, H., An, Y., The application of silk fibroin in tissue engineering and drug carrier area. 2013, 634-638, $1120-1123$.

[5] U. S. National Archives and Records Administration. Natural nonabsorbable silk surgical suture. Code of Federal Regulations 21CFR878.5030, 2006.

[6] Agapov, I. I., Moisenovich, M. M., Druzhinina, T. V., Kamenchuk, Y. A. et al., Biocomposite scaffolds containing regenerated silk fibroin and nanohydroxyapatite for bone tissue regeneration. Dokl. Biochem. Biophys. 2011, 440, 228-230.

[7] Rockwood, D. N., Preda, R. C., Yücel T., Wang, X. et al., Materials fabrication from Bombyx mori silk fibroin. Nat. Protoc. 2011, 6, 1612-1631.

[8] Brenckle, M. A., Tao, H., Kim, S., Paquette, M. et al., Proteinprotein nanoimprinting of silk fibroin films. Adv. Mater. 2013, 25, 2409-2414.

[9] Chutipakdeevong, J., Ruktanonchai, U. R., Supaphol, P., Process optimization of electrospun silk fibroin fiber mat for accelerated wound healing. J. Appl. Polym. Sci. 2013, 130, 3638 3644.

[10] Zhou, C. Z., Confalonieri, F., Jacquet, M., Perasso, R. et al., Silk fibroin: Structural implications of a remarkable amino acid sequence. Proteins 2001, 44, 119-122.

[11] Zhou, C. Z., Confalonieri, F., Medina, N., Zivanovic, Y. et al., Fine organization of Bombyx mori fibroin heavy chain gene. Nucl. Acids Res. 2000, 28, 2418-2419.

[12] Shimanovich, U., Bernardes, G., Knowles, T., Cavaco-Paulo, A., Protein micro- and nano-capsules for biomedical applications. Chem. Commun. 2014, 43, 1361-1371.

[13] Tudora, M., Zaharia, C., Stancu, I., Vasile, E. et al., Natural silk fibroin micro- and nanoparticles with potential uses in drug delivery systems. UPB Scientific Bulletin, Series B: Chemistry and Materials Science 2013, 75, 43-52.

[14] Srihanam, P., Srisuwan, Y., Imsombut, T., Baimark, Y., Silk fibroin microspheres prepared by the water-in-oil emulsion solvent diffusion method for protein delivery. Korean J. Chem. Eng. 2011, 28, 293-297.
[15] Pathak, Y., Thassu, D., Drug Delivery Nanoparticles Formulation and Characterization, Informa Healthcare, New York, USA 2009.

[16] Sundar, S., Kundu, J., Kundu, S., Biopolymeric nanoparticles. Sci. Technol. Adv. Mater. 2010, 11, 1-13.

[17] Lovelyn, C., Attama, A., Current state of nanoemulsions in drug delivery. J. Biomater. Nanobiotechnol. 2011, 2, 626639.

[18] Mohanraj, V., Chen, Y., Nanoparticles - A review. Trop. J. Pharm. Res. 2006, 5, 561-573.

[19] Mason, T., Graves, S., Wilking, J., Lin, M., Extreme emulsification: Formation and structure of nanoemulsions. Condens. Matter Phys. 2006, 9, 193-199.

[20] Mason, T., Wilking, J., Meleson, K., Chang, C. et al., Nanoemulsions: Formation, structure, and physical properties. J. Phys. Condens. Matter 2006, 18, R635-R666.

[21] Silva, R., Ferreira, H., Azoia, N., Shimanovich, U. et al., Insights on the mechanism of formation of protein microspheres in biphasic system. Mol. Pharm. 2012, 9, 3079-3088.

[22] Silva, R., Ferreira, H., Carvalho, A. C., Gomes, A. C. et al., Protein microspheres as suitable devices for piroxicam release. Colloids Surf. B Biointerfaces 2012, 92, 277-285.

[23] Silva, R., Ferreira, H., Vasconcelos, A., Gomes, A. C. et al., Sonochemical proteinaceous microspheres for wound healing. Adv. Exp. Med. Biology 2012, 733, 155-164.

[24] Tadros, T., Colloid aspects of cosmetic formulations with particular reference to polymeric surfactants, in: Tadros, T. (Ed.), Colloids in Cosmetics and Personal Care, Wiley-VCH Verlag, Weinheim, Germany 2008.

[25] Dumortier, G., Grossiord, J. L., Agnely, F., Chaumeil, J. C., A review of poloxamer 407 pharmaceutical and pharmacological characteristics. Pharm. Res. 2006, 23, 2709-2728.

[26] Kim, S., Fernandes, M., Matamá, T., Loureiro, A. et al., Chitosan-lignosulfonates sono-chemically prepared nanoparticles: Characterization and potential applications. Colloids Surf. B Biointerfaces 2013, 103, 1-8.

[27] Kloet, J., Schramm, L., The effect of shear and oil/water ratio on the required hydrophile-lipophile balance for emulsification. J. Surfactants Deterg. 2002, 5, 19-24.

[28] Qiang, C., McClements, D., Formation of nanoemulsions stabilized by model food-grade emulsifiers using high-pressure homogenization: Factors affecting particle size. Food Hydrocoll. 2011, 25, 1000-1008.

[29] Wu, X., Hou, J., Li, M., Wang, J. et al., Sodium dodecyl sulfateinduced rapid gelation of silk fibroin. Acta Biomater. 2012, 8, 2785-2192.

[30] Lo, Y., Relationships between the hydrophilic-lipophilic balance values of pharmaceutical excipients and their multidrug resistance modulating effect in Caco-2 cells and rat intestines. J. Control. Release 2003, 90, 37-48.

[31] Mura, P., Faucci, M., Bramanti, G., Corti, P., Evaluation of transcutol as a clonazepam transdermal permeation enhancer from hydrophilic gel formulations. Eur. J. Pharm. Sci. 2000, 9, 365-372.

[32] Mandal, S., Mandal, S., Microemulsion drug delivery system: A platform for improving dissolution rate of Poorlt water soluble drug. Int. J. Pharm. Sci. Nanotechnol. 2011, 3, 12141219. 


\section{Engineering}

www.els-journal.com in Life Sciences
[33] Solanki, S., Sarkar, B., Dhanwani, R., Microemulsion drug delivery system: For bioavailability enhancement of ampelopsin. ISRN Pharm. 2012, 2012, 1-4.

[34] DeLauder, S., Kidwell, D., The incorporation of dyes into hair as a model for drug binding. Forensic Sci. Int. 2000, 107, 93-104.

[35] Shu, X., Zhu, K., A novel approach to prepare tripolyphosphate: Chitosan complex beads for controlled release drug delivery. Int. J. Pharm. 2000, 201, 51-58.

[36] Teichmann, A., Heuschkel, S., Jacobi, U., Presse, G. et al., Comparison of stratum corneum penetration and localization of a lipophilic model drug applied in an o/w microemulsion and an amphiphilic cream. Eur. J. Pharm. Biopharm. 2007, 67, 699706.

[37] Wu, B., Borland, S., Giordano, R., Cima, L. et al., Solid freeform fabrication of drug delivery devices. J. Control. Release 1996, 40, 77-87.

[38] Uddin, K., Hossain, S., A comparative study on silk dyeing with acid dye and reactive dye. Int. J. Eng. Technol. 2010, 10, 21-26.

[39] Wang, H., Han, J., Feng, X., Pang, Y., Study of inclusion complex formation between tropaeolin $\mathrm{OO}$ and $\beta$-cyclodextrin by spectrophotometry and infrared spectroscopy. Spectrochim. Acta A Mol. Biol. 2006, 65, 100-105.

[40] Sah, M. K., Kumar, A., Pramanik, K., The extraction of fibroin protein from Bombyx mori silk cocoon: Optimization of process parameters. Int. J. Bioinform. Res. 2010, 2, 33-41.

[41] Wray, L. S., Hu, X., Gallego, J., Georgakoudi, I. et al., Effect of processing on silk-based biomaterials: Reproducibility and biocompatibility. J. Biomed. Mater. Res. B Appl. Biomater. 2011, 99, 89-101.

[42] Hess, B., Kutzner, C., Spoel, D., Lindahl, E., GROMACS 4: Algorithms for highly efficient, load-balanced, and scalable molecular simulation. J. Chem. Theor. Comput. 2008, 4, 435447.

[43] Hess, B., Bekker, H., Berendsen, H. J. C., Fraaije, J., LINCS: A linear constraint solver for molecular simulations. J. Comput. Chem. 1997, 18, 1463-1472.

[44] Miyamoto, S., Kollman, P., Settle: An analytical version of the SHAKE and RATTLE algorithm for rigid water models. J. Comput. Chem. 1992, 13, 952-962.

[45] Smith, P., Gunsteren, W., Consistent dielectric properties of the simple point charge and extended simple point charge water models at 277 and 300 K. J. Chem. Phys. 1994, 100, 3169.
[46] Hermans, J., Berendsen, H., Gunsteren, W., Postma, J., A consistent empirical potential for water-protein interactions. Biopolymers 1984, 23, 1513-1518.

[47] Berendsen, H., Postma, J., Gunsteren, W., DiNola, A. et al., Molecular dynamics with coupling to an external bath. J. Chem. Phys. 1984, 81, 3684.

[48] Bussi, G., Donadio, D., Parrinello, M., Canonical sampling through velocity-rescaling. J. Chem. Phys. 2007, 126, 014101.

[49] Grebler, S., Gazso, A., Simkó, M., Friedeler, U. et al., Nanotechnology in cosmetics. NanoTrust Dossiers 2010, 008en, 1-6.

[50] Barth, A., Zscherp, C., What vibrations tell us about proteins. Q. Rev. Biophys. 2002, 35, 369-430.

[51] Kong, J., Shaoning, Y., Fourier tranform infrared spectroscopic analysis of protein secondary structures. Acta Biochim. Biophys. Sinica 2007, 39, 549-559.

[52] Rabotyagova, O., Cebe, P., Kaplan, D., Role of polyalanine domains in $\beta$-sheet formation in spider silk block copolymers. Macromol. Biosci. 2010, 10, 49-59.

[53] Zhao, Z., Li, Y., Chen, A., Zheng, Z. et al., Generation of silk fibroin nanoparticles via solution-enhanced dispersion by supercritical $\mathrm{CO}_{2}$. Indus. Eng. Chem. Res. 2013, 52, 3752-3761.

[54] Wang, X., Wenk, E., Matsumoto, A., Meinel, L. et al., Silk microspheres for encapsulation and controlled release. J. Control. Release 2007, 117, 360-370.

[55] Greenwood, R., Kendall, K., Selection of suitable dispersants for aqueous suspensions of zirconia and titania powders using acoustophoresis. J. Eur. Ceram. Soc. 1999, 19, 479-488.

[56] Hanaor, D., Michelazzi, M., Leonelli, C., Sorrell, C., The effects of carboxylic acids on the aqueous dispersion and electrophoretic deposition of $\mathrm{ZrO}_{2}$. J. Eur. Ceram. Soc. 2012, 32, 235-244.

[57] Riddick, T., Control of Colloid Stability through Zeta Potential, Zeta-Meter, Livingston, New York 1968.

[58] Salopek, B., Krasic, D., Filipovic, S., Measurement and application of zeta-potential. RGN Zbornik 1992, 4, 147-151.

[59] Nogueira, E., Loureiro, A., Nogueira, P., Freitas, J. et al., Liposome and protein based stealth. Chem. Commun. 2013, 166, 417-429.

[60] Xiao, S., Wang, Z., Ma, H., Yang, H. et al., Effective removal of dyes from aqueous solution using ulrafine silk fibroin powder. Adv. Powder Technol. 2014, 25, 574-581.

[61] Matsumoto, A., Chen, J., Collette, A., Kim, U. et al., Mechanisms of silk fibroin sol - gel transitions. J. Phys. Chem. B 2006, 110, 21630-21638. 